BRX TH-635

CALT 68-2825

\title{
No Bel-Robinson Tensor for Quadratic Curvature Theories
}

\author{
S. Deser \\ Physics Department, Brandeis University, Waltham, MA 02454 and \\ Lauritsen Laboratory, California Institute of Technology, Pasadena, CA 91125 \\ deser@brandeis.edu \\ J. Franklin \\ Reed College, Portland, OR 97202 \\ jfrankli@reed.edu
}

\begin{abstract}
We attempt to generalize the familiar covariantly conserved Bel-Robinson tensor $B_{\mu \nu \alpha \beta} \sim$ $R R$ of GR and its recent topologically massive third derivative order counterpart $B \sim R D R$, to quadratic curvature actions. Two very different models of current interest are examined: fourth order $D=3$ "new massive", and second order $D>4$ Lanczos-Lovelock, gravity. On dimensional grounds, the candidates here become $B \sim D R D R+R R R$. For the $D=3$ model, there indeed exist conserved $B \sim \partial R \partial R$ in the linearized limit. However, despite a plethora of available cubic terms, $B$ cannot be extended to the full theory. The $D>4$ models are not even linearizable about flat space, since their field equations are quadratic in curvature; they also have no viable $B$, a fact that persists even if one includes cosmological or Einstein terms to allow linearization about the resulting $\mathrm{dS}$ vacua. These results are an unexpected, if hardly unique, example of linearization instability.
\end{abstract}

\section{Introduction}

The Bel-Robinson tensor $B_{\mu \nu \alpha \beta}$ was first discovered in ordinary Einstein gravity (GR) at $D=4$, in a search for a gravitational counterpart of the usual matter stress-tensor $T_{\mu \nu}$. Since there can be neither local tensorial gravitational candidates of second derivative order (because quantities $\sim \partial g_{\mu \nu} \partial g_{\alpha \beta}$, being frame-dependent, can be made to vanish at any point), nor any non-covariantly conserved ones, the successful candidate was indeed quadratic in the tensorial "field strengths" curvatures $B \sim R R$, and covariantly conserved, in analogy with the quadratic Maxwell $T_{\mu \nu} \sim F F$. Subsequently, conserved $B$ were found for arbitrary dimension and matter analogs have also been constructed (see, e.g., [1] for earlier references).

Very recently, we showed that a conserved $B \sim R D R$ could be defined for topologically massive gravity [2]. Given this theory's third derivative order, we speculated there on extending $B$ 
to other gravitational systems, in particular to the currently popular quadratic curvature models. The present note reports a negative outcome in two active, very different theories. For the fourth derivative $D=3$ new massive gravity (NMG) [3], without an Einstein term [4] for simplicity, there is a $B \sim \partial R \partial R$ at linearized level but it cannot be extended to the full theory, despite an enormous number of possible cubic correction terms. The Lanczos-Lovelock (LL) [5] models' quadratic curvature field equations, $\sim R R=0$, obviously cannot even be linearized about flat space and turn out to have no conserved $B$ either. Adding cosmological or Einstein terms to permit linearization (to effective cosmological GR) about the resulting $\mathrm{dS}$ vacua only allows the usual linear $B \sim R R$ of GR, but no nonlinear extension.

\section{Fourth Order Models}

Since the machinery is considerably more complicated here than in GR, we analyze the (purely quadratic part of) NMG [3]:

$$
I=\int d^{3} x \sqrt{-g}\left(\bar{R}^{\mu \nu} \bar{R}_{\mu \nu}-\bar{R}^{2}\right), \quad \bar{R}_{\mu \nu} \equiv R_{\mu \nu}-\frac{1}{4} g_{\mu \nu} R .
$$

[The Cotton tensor of TMG is the curl of the Schouten tensor $\bar{R}_{\mu \nu}$.] The resulting field equations,

$$
\square \bar{R}_{\mu \nu}-D_{\mu} D_{\nu} \bar{R}-4 \bar{R}_{\mu}{ }^{\sigma} \bar{R}_{\nu \sigma}+\bar{R} \bar{R}_{\mu \nu}+g_{\mu \nu} \bar{R}^{2}=0
$$

of course obey Bianchi identities since (1) is an invariant. The field equations begin as $D D \bar{R} \sim 0$, so $B$ cannot just imitate the $R R$ of GR, but must instead start as $B \sim D \bar{R} D \bar{R}$, along with possible cubic, $\bar{R} \bar{R} \bar{R}$, terms since they have the same dimension as $D \bar{R} D \bar{R}$. Schematically then, we expect that

$$
B \sim D \bar{R} D \bar{R}+\bar{R} \bar{R} \bar{R} .
$$

[One can also consider similar terms $\sim D(\bar{R} D \bar{R})$ or $\bar{R} D D \bar{R}$, but they are already irrelevant at linear level.] We begin with the linearized (about flat space) truncation, $\partial \partial \bar{R}=0$, of (2); the corresponding linearized $B$ is simply given by

$$
B_{\mu \nu \alpha \beta}=\bar{R}_{\alpha \beta}{ }^{\sigma}\left(\bar{R}_{\sigma \nu, \mu}-\bar{R}_{\mu \nu, \sigma}\right) .
$$

Its conservation,

$$
\partial^{\mu} B_{\mu \nu \alpha \beta}=0
$$

is verified using the field equations, the Bianchi identity, and antisymmetry of the parenthesis in (4). Conservation also holds for all permutations of $B$ 's $(\nu \alpha \beta)$ indices; for completeness, we note also the identically conserved, if irrelevant, $b_{\mu \nu \alpha \beta}=D^{\gamma} H_{[\gamma \mu] \nu \alpha \beta}, H$ antisymmetric in $\gamma \leftrightarrow \mu$.

Thus encouraged, we turn to candidates (3) at nonlinear level, where the now covariant derivatives $D_{\mu}$ no longer commute. At first sight, there are so many available independent terms cubic in $\bar{R}_{\mu \nu}$ and $g_{\mu \nu} \bar{R}$, that success seems guaranteed; however, it turns out to be unattainable. The simplest procedure is to take the covariant divergence of the initial $B \sim D \bar{R}(D \bar{R}-D \bar{R})$ ansatz and try to compensate for the resulting cubic $\sim \bar{R} \bar{R} D \bar{R}$ terms by adding suitable $\bar{R} \bar{R} \bar{R}$ to $B$. The 
procedure is straightforward, using the $D=3$ properties

$$
\begin{aligned}
R_{\mu \nu \rho \sigma} & =g_{\mu \rho} \bar{R}_{\nu \sigma}+g_{\nu \sigma} \bar{R}_{\mu \rho}-g_{\nu \rho} \bar{R}_{\mu \sigma}-g_{\mu \sigma} \bar{R}_{\nu \rho} \\
{\left[D_{\alpha}, D_{\beta}\right] V^{\gamma} } & =\left[\delta_{\beta}^{\gamma} \bar{R}_{\sigma \alpha}-\delta_{\alpha}^{\gamma} \bar{R}_{\sigma \beta}+g_{\sigma \alpha} \bar{R}_{\beta}^{\gamma}-g_{\sigma \beta} \bar{R}_{\alpha}^{\gamma}\right] V^{\sigma} .
\end{aligned}
$$

We find

$$
D^{\mu} B_{\mu \nu \alpha \beta}=\left[\bar{R}^{\rho \mu} \bar{R}_{\rho \beta}\left(\bar{R}_{\mu \nu ; \alpha}-\bar{R}_{\alpha \nu ; \mu}\right)+\frac{1}{2} \bar{R}_{\alpha \beta ; \sigma}\left(\bar{R}^{\sigma \rho} \bar{R}_{\rho \nu}-\bar{R}_{\nu}^{\sigma} \bar{R}\right)\right]+(\alpha \leftrightarrow \beta),
$$

to which could be added the above $(\alpha \beta \nu)$ permutations. The problem is now to find, among all $(\bar{R} \bar{R} \bar{R})_{\mu \nu \alpha \beta}$, those whose $\mu$-divergence cancels $(7)$, or the sum of permutations. Note that $(7)$ contains both $\mu$ - and $\alpha, \beta$-derivatives, and that the latter must arise from cubic terms of the form $\sim g_{\mu \alpha}(\bar{R} \bar{R} \bar{R})_{\nu \beta}$, and possibly $g_{\mu \alpha} g_{\nu \beta}(\bar{R} \bar{R} \bar{R})$; these are also plentiful. This systematic approach failed: any compensating term also created a new problematic one. We then undertook the brute force approach, involving the sum of all dimensionally possible candidate terms, $\sim D \bar{R} D \bar{R}+$ $\bar{R} D D \bar{R}+\bar{R} \bar{R} \bar{R}$; to be sure, any $D \bar{R} D \bar{R}$ combinations that fail the linearized conservation test could be excluded a priori.

We sketch the procedure and counts, but omit the uninstructive, laborious, details 1 . Start with the thirty nine possible terms of the form $\bar{R} \bar{R} \bar{R}$, incuding $g \bar{R} \bar{R} \bar{R}$ terms, together with the 75 $D(\bar{R} D \bar{R})$ candidates, giving a total of $m=114$ potential terms. Take the divergence of each of these, enforcing the on-shell, Bianchi and three-dimensional simplifications. That gives, for each term, a right-hand side that is a linear combination of $(D D \bar{R}) D \bar{R}, \bar{R} \bar{R} D \bar{R}$ and $\bar{R} D D D \bar{R}$. There are $n=231$ unique terms of this form in these sums. We can view the action of $D^{\mu}$ as a linear operator taking the $m$ starting ingredients to a space of $n$ outputs: assign to each candidate term a basis vector $\left\{\mathbf{e}_{i}\right\}_{i=1}^{m}$, and to each term appearing in the sums $D^{\mu} \mathbf{e}_{i}$, the basis vector $\left\{\mathbf{E}_{i}\right\}_{i=1}^{n}$, the matrix $\mathbb{D} \in \mathbb{R}^{n \times m}$ just maps $\mathbf{e}_{k}$ to its sum: $\mathbb{D} \mathbf{e}_{k}=\sum_{i=1}^{n} \alpha_{i} \mathbf{E}_{i}$. We constructed this matrix using a symbolic algebra package, and found that $\mathbb{D}$ has no null space, and so no conserved Bel-Robinson tensor exists.

\section{Second Order, LL, Theories}

At the other end of the quadratic curvature spectrum from the $D=3$ model (1) lie the $D>4$ LL theories, whose field equations remain of second derivative order, but do not admit any linearized expansion about flat space. The Lagrangians are, in vielbein formulation,

$$
L=\epsilon^{a b c d f \cdots} \epsilon^{A B C D F \cdots} R_{a b A B} R_{c d C D} e_{f F} \cdots
$$

The field equations simply consist of removing one vielbein factor,

$$
E^{f F} \equiv(\epsilon \epsilon R R e)^{f F}=0,
$$

\footnotetext{
${ }^{1}$ These may be found in the notebook at: http://people.reed.edu/ jfrankli/NullSpace.nb.
} 
since the curvatures' variations vanish by the cyclic identities. Lowercase indices are local, capitals are world. Diffeomorphism invariance of the action ensures that $D_{F} E^{f F}$ vanishes identically, by the cyclic identities. Indeed, this is even true in $D>5$ : if we open any second pair of indices $(g G)$, there results an identically conserved, hence uninteresting, $H^{f g \ldots F G \ldots}$. We cannot even start with a linearized $B$, since the field equations (9) are intrinsically nonlinear. That there is no true $B$ is rather clear: for example, attaching a curvature to $H$ above loses conservation, which no additional cubics can help, while no $D R D R$ term is relevant just because it is linearizable. Extending LL to include a cosmological or Einstein term does allow for linearization about a nonflat, dS, vacuum: a dS metric ansatz reduces (9) to the equation $\sim a \Lambda^{2}=b \Lambda$ in either choice. There is always a flat, $\Lambda=0$, but also now a $\mathrm{dS}, \Lambda=b / a$, vacuum 2 . About the latter, the system linearizes to cosmological Einstein, which of course enjoys the same conserved $B \sim R R$ as $\Lambda=0$ GR. However, just like its predecessor in NMG, this $B$ evaporates as soon as we go to the full theory, since there is still no way to take into account the nonlinear $R R$ part of (9).

\section{Conclusion}

We conclude that both NMG and Einstein+LL violate Fermi's rule: if the lowest order works, the conjecture is correct, while pure LL doesn't even have a lowest (here linearized) order. Clearly there is no invariance here like that [1] underlying $B$-conservation in GR.

SD acknowledges support from NSF PHY-1064302 and DOE DE-FG02-164 92ER40701 grants.

\section{References}

[1] S. Deser, in "Gravitation and Relativity in General" (ed F. Atrio-Barandela and J. Martins), World Publishing (1999), gr-qc/9901007; S. Deser and Z. Yang, Class. Quant. Grav. 71491 (1990).

[2] S. Deser and J. Franklin, Class. Quant. Grav. 28032002 (2011), arXiv:1011.4240.

[3] E. A. Bergshoeff, O. Hohm and P. K. Townsend, Phys. Rev. Lett. 102201301 (2009), hepth/0901.1766.

[4] S. Deser, Phys. Rev. Lett. 103101302 (2009), hep-th/0904.4473.

[5] C. Lanczos, Ann. Math. 39842 (1938); D. Lovelock, J. Math. Phys. 12498 (1971).

[6] D.G. Boulware and S. Deser, Phys. Rev. Lett. 552656 (1985).

\footnotetext{
${ }^{2}$ While the appearance of a second, dS, vacuum in $R+\mathrm{LL}-$ indeed in all $R+R^{2}$ models but $(D=4) R+$ Weyl ${ }^{2}-$ is reasonably clear [6], it may seem counter-intuitive that, unlike GR, quadratic models with cosmological term allow both flat and dS vacua.
} 